\title{
Atrofia ténar em sindromas de túnel cárpico graves - 21 casos operados
}

\author{
J. C. Botelheiro(1), S. SiLVÉRIo(2), G. Viana ${ }^{(3)}$ \\ Unidade de Cirugia da Mão do Hospital Ortopédico de Sant'Ana, Parede, Portugal \\ ${ }^{(1)}$ Assistente GRADUADo dE ORTOPEDIA, RESPONSÁVEL DA UNIDADE DE CIRURGIA DA MÃo \\ (2)ASSISTENTE DE ORTOPEDIA DA UNIDADE DE CIRURGIA DA MÃO \\ ${ }^{(3)}$ INTERNO DE ORTOPEDIA
}

Correspondencia:

Dr. José Carlos Botelheiro

Hospital de Sant'Ana

Rua de Benguela, 2779-501

Parede, Portugal

Teléf: 214585600

Fax 21 4585601,

e-mail: jcbotelheiro@gmail.com

Introducción: La atrofia tenar en los casos graves de síndrome de túnel carpiano puede causar una pérdida de la oposición o, en casos raros, una inestabilidad radial metacarpo-falángica del pulgar. Los resultados de plastias de oposición y de ligamentoplastias metacarpo-falángica, hechas en el mismo tiempo que la liberación del túnel carpiano, fueron estudiados en 21 pacientes operados.

Material y Método: 21 pacientes fueron operados - 18 plastias de oposición (13 Camitz, 2 Ney y 3 Matev), 2 ligamentoplastias y 1 artrodesis metacarpo-falángica.

Resultados: Las plastias de oposición han dado una oposición activa al tercer dedo en 6 casos, al anular en 6 y al meñique en otros 6 . Las ligamentoplastias y la artrodesis metacarpo-falángicas mejoraron mucho la función del pulgar en los casos de inestabilidad.

Conclusión: Las plastias de oposición y las ligamentoplastias metacarpo-falángicas hechas en el mismo tiempo quirúrgico que la liberación del túnel carpiano mejoraron mucho la pérdida de oposición o la rara inestabilidad radial metacarpo-falángica de algunos casos graves de síndrome de túnel carpiano.

Palabras clave: síndrome túnel carpiano, oponentoplastia, ligamentoplastia
Objective: Thenar muscle atrophy in severe cases of tunnel carpal syndrome can cause a loss of opposition or, rarely, a radial metacarpophalangeal thumb instability. Results of some techniques of opponensplasty or metacarpophalangeal ligamentoplasty done at the same time of the median nerve decompression were studied in 21 operated patients. Material and method: 21 patients were operated - 18 opponensplasties (13 Camitz, 2 Ney and 3 Matev), 2 metacarpophalangeal ligamentoplasties and 1 arthrodesis.

Results: Opponensplasties gave an active opposition to the long finger in 6 patients to the ring finger in 6 and to the auricular in 6. Metacarpophalangeal ligamentoplasties and the arthrodesis clearly improved the thumb function in cases of metacarpophalangeal instability.

Conclusion: Opponensplasties or metacarpophalangeal ligamentoplasties done at the same time of neurolysis greatly improve the loss of opposition or the rare metacarpophalangeal thumb instability of severe cases of carpal tunnel syndrome.

Key words: tunel carpal sydrome, opponnensplasties, ligamentoplasties

Rev. Iberam. Cir. Mano - Vol. 35 • Núm. 2 Noviembre 2007 (22-26) 


\section{INTRODUCÃO}

$\mathrm{N}$ os sindromas de túnel cárpico graves pode haver uma maior ou menor atrofia dos músculos inervados pelo ramo motor ténar do nervo mediano - abductor polilcis brevis (APB), opponens pollicis e parte ou totalidade do flexor pollicis brevis.

Conforme a extensão da inervação desse ramo e a gravidade da atrofia pode haver ou não parésia ou paralisia da oponência do polegar, que geralmente não melhora após a neurolise, até pela idade dos doentes (Figura 1), embora alguns autores refiram uma boa recuperação muscular (mas não da oponência) após a simples abertura do túnel cárpico ${ }^{1,2}$.

Havendo parésia grave ou paralisia da oponência parece assim que deveria ser feita uma oponentoplastia e neurolise do mediano no mesmo tempo ${ }^{3}$.

As oponentoplastias cujo tendão cruza por cima do nervo mediano libertado devem teoricamente ser evitadas pelo risco de nova compressão deste ${ }^{4}$.

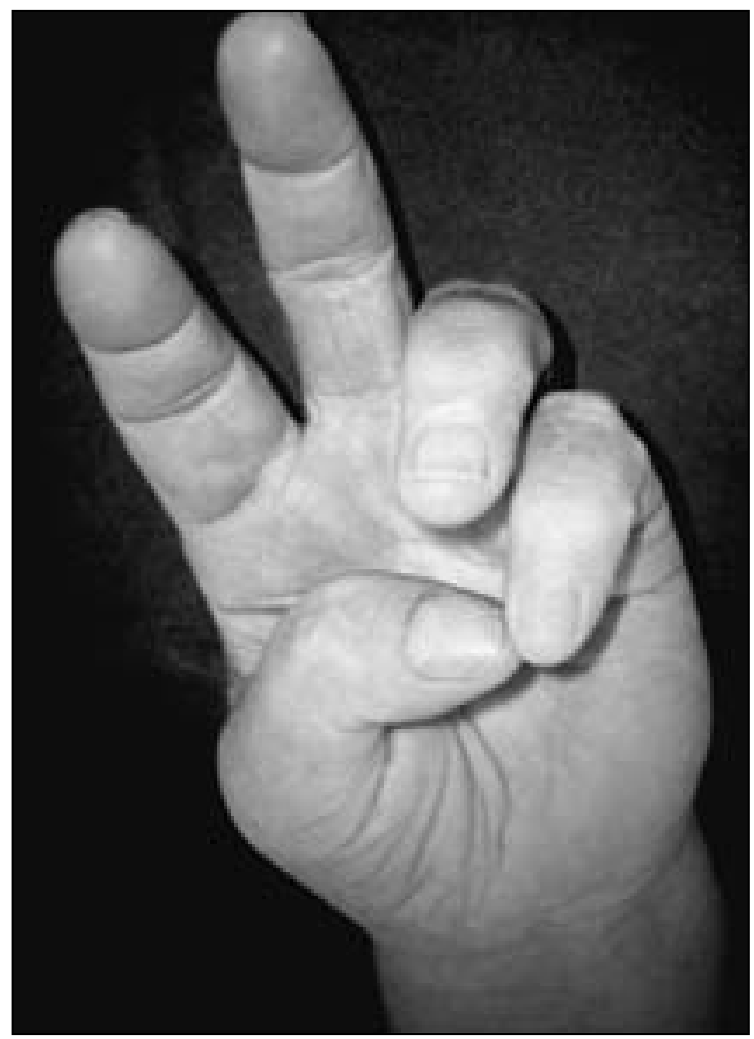

Figura 1: Sindroma de tunel cárpico grave com oponência muito fraca.
A oponentoplastia de Camitz ${ }^{5}$, em que o tendão do palmaris longus (PL) é prolongado por uma tira de fascia palmar que é suturada ao tendão terminal do APB, é simples, elegante e dá resultados aceitáveis com uma recuperação rápida $^{6}$, talvez por o PL ser sinérgico com a oponência (Figura 2).

Se a fascia palmar for insuficiente ou em casos de neurólise previa pode ser utilizada a técnica de $\mathrm{Ney}^{7}$ modificada, com o PL suturado ao tendão do extensor pollicis brevis (EPB), cortado proximalmente e desviado da sua goteira ${ }^{8}$.

Se não existir PL pode ser feita uma oponentoplastia com o EPB - por exemplo a de Matev $^{9}$ - embora Foucher ${ }^{4}$ aconselhe, nesses casos, a fazer uma oponentoplastia clássica num segundo tempo operatório, quando houver já uma fibrose cicatricial que impeça a compressão do mediano pelo tendão transposto.

Por outro lado, tivemos a oportunidade de observar também alguns doentes com sindromas de túnel cárpico graves, com grande atrofia ténar mas com boa oponência activa, nos quais no entanto a função do polegar estava mui-

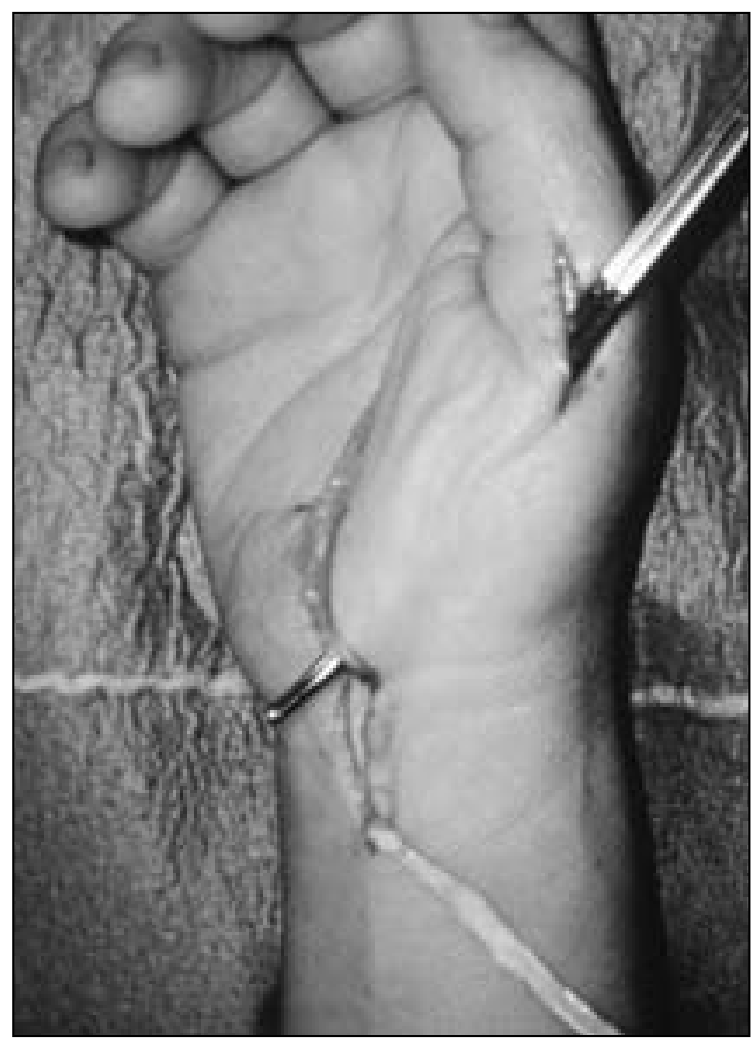

Figura 2: Oponentoplastia de Camitz - imagem intraoperatória. 


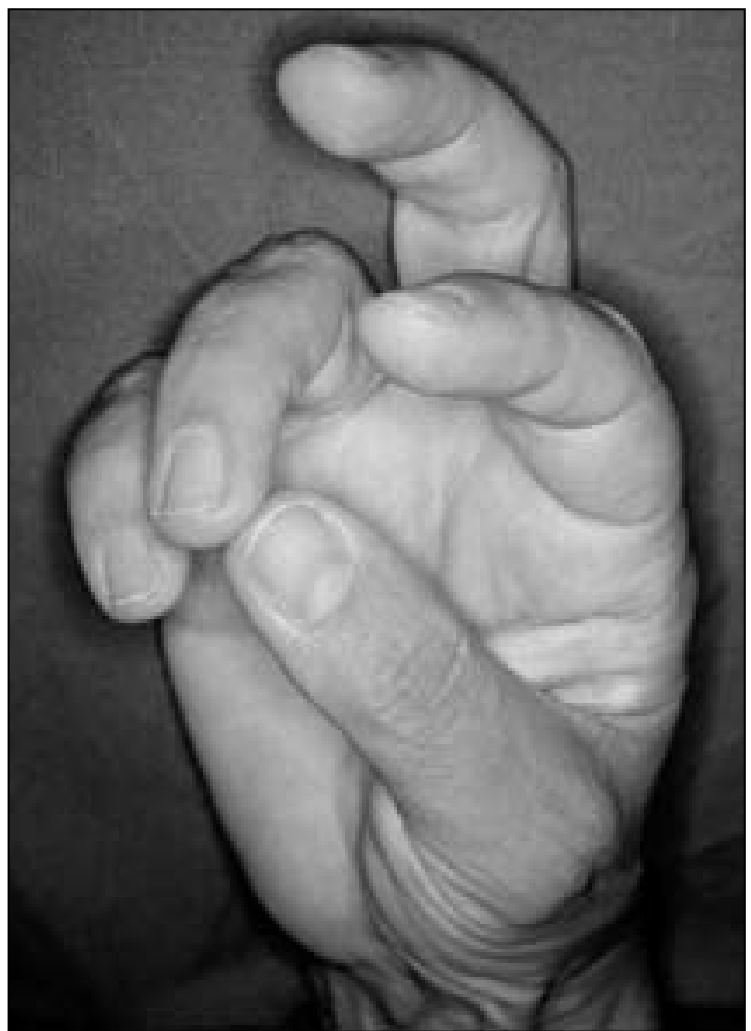

Figura 3: Oponentoplastia de Camitz - resultado posoperatório.

to diminuida por uma grave instabilidade metacarpo-falângica (MCF) radial, sem antecedente traumático, que atribuimos à atrofia do APB. Nestes casos, não descritos na literatura médica que conhecemos, fizemos uma ligamentoplastia em dois e uma artrodese num terceiro.

O propósito deste trabalho é expôr a nossa experiência deste assunto, descrevendo as diferentes técnicas cirúrgicas utilizadas para restabelecer a oponência, suas indicações, resultados e complicações, para o que revimos os processos dos 21 doentes operados.

\section{MATERIAL E MÉTODO}

Reviram-se retrospectivamente 21 casos consecutivos de atrofia ténar grave secundária a sindromas de túnel cárpico operados nos últimos 15 anos na Unidade de Cirurgia da Mão do Hospital Ortopédico de Sant'Ana pelos dois primeiros autores do trabalho.

Dos 21 casos, 20 eram do sexo feminino e 17 do lado direito. Tinham uma média de ida-

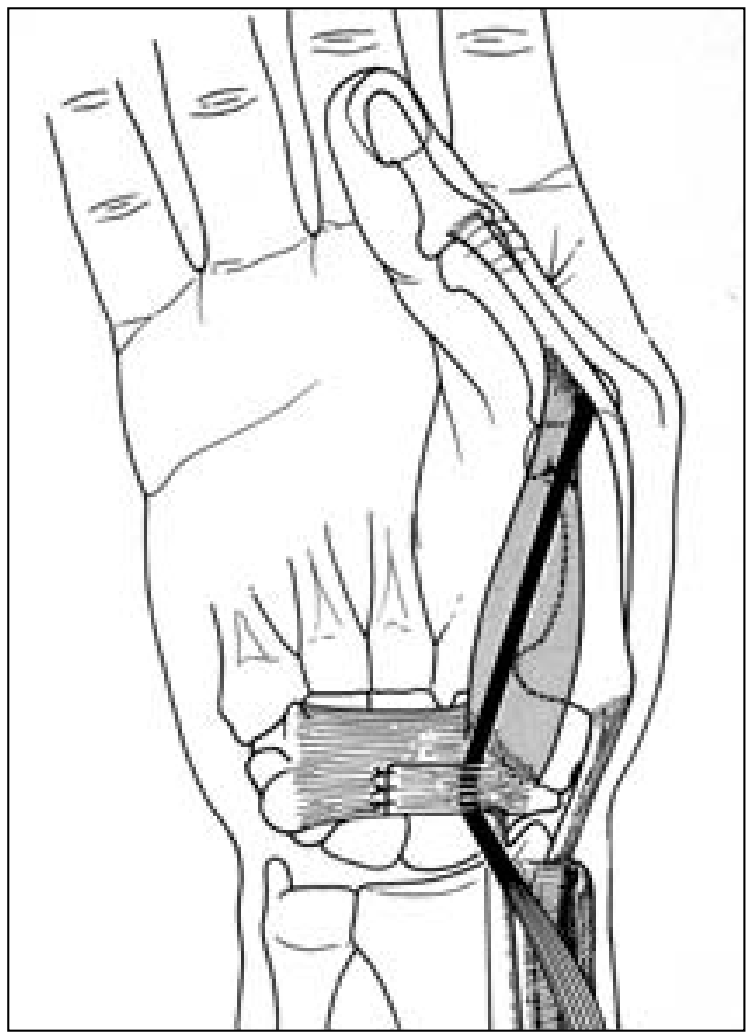

Figura 4: Oponentoplastia de Matev.

de de 55 (rango, 9-73), sendo que a doente mais jovem tinha provavelmente uma doenca metabólica de Weil-Marchesani.

De notar que antes da cirurgia nenhum doente tinha uma verdadeira oponência «polpa a polpa» sequer ao indicador.

Das 18 doentes em que fizemos oponentoplastias, em 13 utilizamos a clássica técnica de Camitz e em 2 a de Ney (1 caso já operado e 1 com uma fascia muito fina). Os doentes de Camitz foram imobilizados com uma tala gessada com o polegar em oponência durante 4 semanas e geralmente não necessitaram de fisioterapia.

Não descrevemos pormenorizadamente as técnicas cirúrgicas das oponentoplastias que se encontram bem descritas em qualquer livro de cirurgia da mão ou nos artigos citados na bibliografia. De notar no entanto que na técnica de Camitz ou na de Ney a incisão na palma da mão deve ser encerrada antes da sutura final da oponentoplastia no polegar.

Em 3 casos sem palmaris longus optámos por uma variante pessoal da técnica de $\mathrm{Matev}^{10}$, 


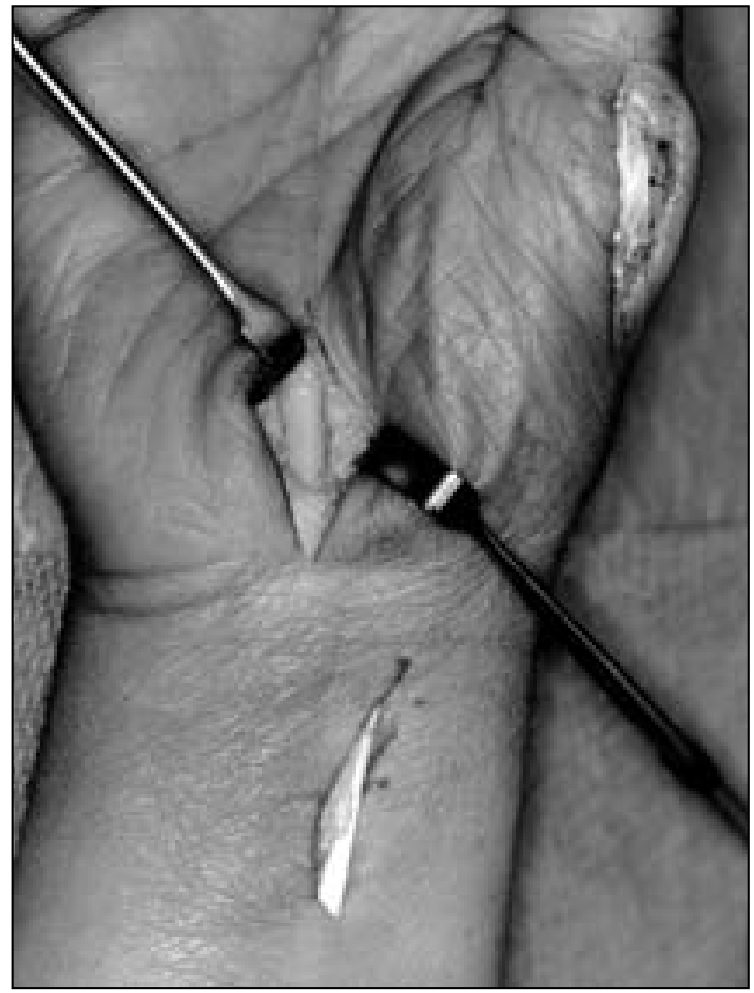

Figura 5: Instabilidade radial MCF corrigida com ligamentoplastia simultânea com libertação do tunel cárpico.

na qual o tendão do EPB, libertado da sua goteira, é desviado cubitalmente por uma «argola» feita com metade da largura do tendão do flexor carpi radialis (FCR), enquanto que na técnica original essa argola era feita à custa do retináculo dos flexores (Figura 4), que nestes casos cortamos para abrir o túnel cárpico. Como o tendão da oponentoplastia não é cortado nem suturado, foram imobilizados apenas 3 semanas, mas necessitaram fisisoterapia para recuperar a oponência.

Em 2 doentes com instabilidade radial MCF do polegar incapacitante optámos por fazer, além da neurolise do mediano, uma plicatura da capsula radial MCF reforçada por uma ligamentoplastia com uma tira de metade da largura do FCR suturada a 2 ancoras ósseas, uma no metacarpico e outra na falange ${ }^{11}$ (Figura 5). Foram imobilizados, com tala gessada e fio de Kirschner transarticular, também durante 4 semanas.

Num terceiro doente, que tinha também uma rizartrose indolora e em que a instabilidade, além de radial, era também palmar, optámos por uma artrodese MCF com «hauban», sem imobilização post-operatória.

\section{RESULTADOS}

Resultados globais das oponentoplastias:

Oponência a D3 - 6 casos

$$
\begin{array}{ll}
\ll \quad & \text { a D4 - } 6 \text { casos } \\
& \text { a D5 - } 6 \text { casos (2 à polpa, } 2 \text { a } \\
& \text { F2, } 2 \text { à base do dedo) }
\end{array}
$$

As ligamentoplastias radiais MCF resultaram numa articulação estável, com perda mínima da mobilidade e uma melhoria notável da função do polegar. A artrodese MCF do polegar melhorou também muito a sua funcionalidade, por exemplo na escrita, apesar da perda de mobilidade.

\section{COMPLICAÇÕES}

Uma das doentes em que utilizámos a técnica de Ney desenvolveu 3 anos depois um quadro doloroso muito intenso no trajecto do tendão transposto, que não melhorou com tratamento conservador, pelo que a reoperámos excisão da oponentoplastia efectuada e sua substituição por uma outra, de Thompson ${ }^{12}$, com o tendão do flexor digitorum sublimis do anelar. Deixou de ter dores e manteve a oponência, sem sintomas de recidiva da compressão do nervo mediano.

\section{DISCUSSÃO}

Este trabalho é uma mera revisão retrospectiva dos doentes operados na nossa Unidade de Cirurgia da Mão, sem pretensões estatísticas mas apenas para apresentar as indicações que utilizámos para as diferentes técnicas e os resultados e complicações que tivemos nesta curta série de doentes operados com esta patologia que, devido á vulgarização das cirurgias do sindroma de túnel cárpico nos países desenvolvidos, provavelmente se irá tornando cada vez mais rara.

\section{CONCLUSÕES}

Nos casos de sindroma do tunel cárpico graves, com atrofia ténar e paralisia ou paré- 
sia da oponência, deve ser feita uma oponentoplastia que não cruze sobre a neurolise do mediano. Além da clássica tecnica de Camitz podem também ser utilizadas a de Ney (quando a fascia palmar não for utilizável) ou de Matev (com o EPB, quando não existir PL).
Nalguns casos raros com uma atrofia ténar grave a oponência é normal mas existe uma instabilidade radial MCF do polegar incapacitante, causada por essa atrofia. Melhoram muito com uma ligamentoplastia radial MCF do polegar, além da neurolise do mediano, devendo o recurso à artrodese MCF ser excepcional.

\section{BIBLIOGRAFÍA}

1. Gelberman RH, Pfeffer GB, Galbraith RT, et al. Results of treatment of severe carpal-tunnel syndrome without internal neurolysis of the median nerve. $\mathrm{J}$ Bone Joint Surg Am, 1987; 896-9.

2. Nolan WB, Alkaitis D, Glickel SZ, et al. Results of treatment of severe carpal tunnel syndrome. J Hand Surg Am, 1992; 17: 1020-2.

3. Davis RC, Barton J. Median nerve palsy. En: Green PD, Hotchkiss RN y Pederson WC (Eds.) Green's Operative Hand Surgery. $4^{a}$ ed. New York: Churchill Livingstone, 1999: 1497525.

4. Foucher G. Nerve entrapment at the wrist level. En: Duparc J.
(Ed.) Surgical Techniques in Orthopaedics and Traumatology, Vol. 5 - Wrist and Hand. Paris: EFFORT - Elsevier, 2002: 55-300-B-10.

5. Camitz H. Ueber die be Handlung der Oppositionslähmung. Acta Orthop Scand, 1929: 65: 77-81.

6. Foucher G, Malizos C, Sammut $D$, et al. Primary palmaris longus transfer as an opponensplasty in carpal tunnel release. A series of 73 cases. J Hand Surg Br, 1991; 16: 56-60.

7. Ney KW. A tendon transplant for intrinsic hand muscle paralysis. Surg Gynecol Obstet, 1921: 33: 342-8.

8. Revol M, Servant JM. Paralysies de la Main et du Membre Su- périeur. Analyse - Principes therapeutiques. Paris: MEDSI, 1987: 128.

9. Matev IB. Restoration of thumb opposition by displacement of extensor pollicis brevis. Hand, 1981: 13: 279-84.

10. Tubiana R. Paralysies motrices du Pouce. En Traité de Chirurgie de la Main, Vol 4. Paris: Masson, 1991: 211-60.

11. McCall TE, Roth MZ. Simple method for repair of collateral ligament injuries of the thumb metacarpophalangeal joint. Plast Reconstr Surg, 2000; 106: 657-9.

12. Thompson TC. A modified operation for opponens paralysis. J Bone Joint Surg, 1942; 24: 632-40. 